\title{
Thermal Properties of LARP Type Nb3Sn Superconducting Magnet Coil Electrical Insulation in Pressurized Superfluid Helium
}

\author{
J. Polinski • P. Bogdan • M. Chorowski • J. Niechcial • \\ A. Noga $\cdot$ P. Pyrka
}

Received: 10 June 2014 / Accepted: 15 September 2014 / Published online: 31 October 2014

(C) The Author(s) 2014. This article is published with open access at Springerlink.com

\begin{abstract}
The development of new generation, highperformance devices, such as MRI scanners or high-energy accelerators, (may be attributed to the use of) Nb3Sn based superconducting (SC) magnets, which are characterized by their ability to produce much higher magnetic fields than the presently used NbTi-based magnets. Due to the wind-andreact technology of the production of Nb3Sn magnets, new methods and materials for the magnet coil electrical insulation are required. The proper design of SC magnets demands the characterization of thermal properties of the new insulation materials, inter alia, in superfluid helium conditions. This paper provides experimental data of the thermal conductivity and Kapitza resistance for S-glass fiber reinforced ceramic-epoxy (LARP type) electrical insulation, measured in pressurized (at 1 bar pressure) superfluid helium in a temperature range of $1.50-2.00 \mathrm{~K}$.
\end{abstract}

Keywords Kapitza resistance - Thermal conductivity · Superfluid helium $\cdot \mathrm{Nb} 3 \mathrm{Sn}$ magnets electric insulation

\section{Introduction}

One of the key elements of the superconducting magnets is magnet coil electrical insulation. The insulation material, aside from high electrical strength, should also be characterized by potentially high mechanical strength in order to withstand the Lorenz forces occurring in the powered magnets. It should also possess the high capacity to transfer

J. Polinski $(\varangle) \cdot$ P. Bogdan · M. Chorowski · J. Niechcial ·

A. Noga $\cdot$ P. Pyrka

Wroclaw University of Technology, Wyb. Wyspianskiego 27,

50-370 Wroclaw, Poland

e-mail: jaroslaw.polinski@pwr.edu.pl heat from the magnet coil to the magnet coolant. Those requirements are fully complied by Kapton polyimide commonly used in the NbTi magnets. Nevertheless, due to the application temperature of Kapton at up to $400{ }^{\circ} \mathrm{C}$, this material is not suitable for the $\mathrm{Nb} 3 \mathrm{Sn}$ magnet wind-andreact production technique, which requires a heat treatment of approximately $650{ }^{\circ} \mathrm{C}$. Therefore, for the further development of Nb3Sn-based technology, new materials and methods for magnet coil electrical insulation are required.

Within the European Coordination for Accelerator Research and Development (EuCARD) program, four potential materials for $\mathrm{Nb} 3 \mathrm{Sn}$ magnets coil electrical insulation have been selected and thermally characterized in pressurized superfluid helium (HeIIp) conditions. The thermal conductivity and Kapitsa resistance for Sglass fiber reinforced epoxies (RAL Mix 71, RAL Mix 237), as well as for cyanate ester epoxy resin (CE epoxy) have already been published $[1,2]$. Here, the data for S-glass-ceramic-epoxy laminate (LARP type) are presented.

\section{Test Procedure}

\subsection{Test Method Principle}

The thermal properties of the tested material have been determined by the so-called "drum method", as schematically presented in Fig. 1. In this method, the tested material sample with surface $A\left[\mathrm{~m}^{2}\right]$ and thickness $L[\mathrm{~m}]$ separates a space into two volumes, both filled with HeIIp. The temperature of the first volume $T_{b}[\mathrm{~K}]$ is controlled at a constant value, while in the second volume, a constant heat $Q_{s}[\mathrm{~W}]$ is dissipated and the temperature $T_{i}[\mathrm{~K}]$ of this volume is measured. 


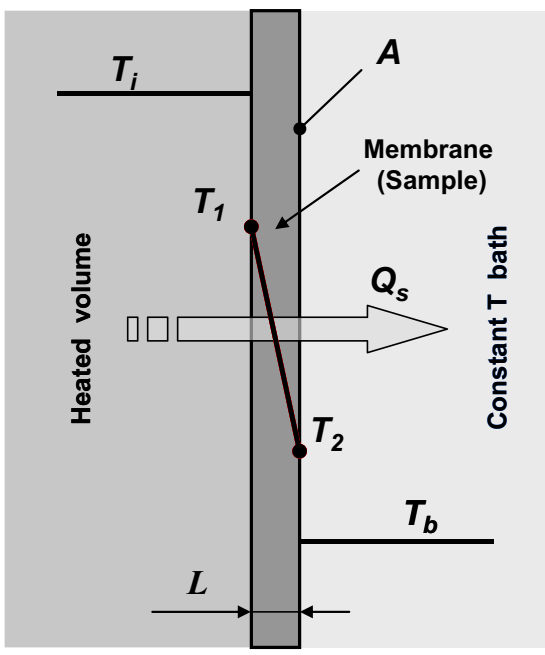

Fig. 1 Drum method scheme

In the considered case, the heat $Q_{\mathrm{s}}$ is transferred between the separated volumes by Kapitza conductance from the heated volume to one surface of the sample. It then goes through the sample material via heat conduction, and then via Kapitza conductance from the second sample surface to the isothermal (constant $T_{\mathrm{b}}$ ) volume. This may be expressed as follows:

$\frac{Q_{\mathrm{s}}}{A}=h_{k}\left(T_{i}\right) \cdot\left(T_{i}^{n}-T_{1}^{n}\right)=\frac{k}{L}\left(T_{1}-T_{2}\right)=h_{k}\left(T_{b}\right) \cdot\left(T_{2}^{n}-T_{b}^{n}\right)$

where $h_{k}$ is the Kapitza coefficient $\left[\mathrm{W} / \mathrm{m}^{2} K^{n}\right]$, and $n$ is the dimensionless Kapitza heat transfer exponent, while $k$ is the heat conduction coefficient of the sample material [W/mK], and $T_{1}$ and $T_{2}[\mathrm{~K}]$ are the temperatures of the sample surface from the heated volume and the isothermal volume side, respectively.

For very small temperature differences between the separated volumes $\left(T_{i}-T_{b}=\Delta T<T_{b}\right)$, the Kapitza conductance can be linearized, and (1) can be written in the following form:

$$
\begin{aligned}
\frac{Q_{s}}{A} & =n \cdot h_{k}\left(T_{i}\right) \cdot T_{i}^{n-1}\left(T_{i}-T_{1}\right)=\frac{k}{L}\left(T_{1}-T_{2}\right) \\
& =n \cdot h_{k}\left(T_{b}\right) \cdot T_{b}^{n-1}\left(T_{2}-T_{b}\right)
\end{aligned}
$$

As $\Delta T<<T_{b}$, it can be considered that $T_{i} \approx T_{b}$, and:

$$
\begin{aligned}
R_{s}=\frac{A \cdot \Delta T}{Q_{s}} & =\frac{1}{n \cdot h_{k}\left(T_{i}\right) \cdot T_{i}^{n-1}}+\frac{L}{k}+\frac{1}{n \cdot h_{k}\left(T_{b}\right) \cdot T_{b}^{n-1}} \\
& =\frac{2}{n \cdot h_{k} \cdot T_{b}^{n-1}}+\frac{L}{k}=2 R_{K}+R_{C}
\end{aligned}
$$

where $R_{S}\left[m^{2} \mathrm{~K} / \mathrm{W}\right]$, is the total thermal resistance of the heat transfer through the tested sample. It can be found from (3) that for small $\Delta T$, the total thermal resistance is the sum of twice the Kapitza resistance $R_{K}$ and the solid conduction resistance $R_{C}$.

\subsection{The Sample Holder}

The sample holder (see the schematic construction in Fig. 2) consists of a central flange and two sets of sample and compression flanges screwed to each side of the central flange. All flanges are made of stainless steel. Inside the central flange, an electrical heater (ceramic resistor) and a temperature sensor are located. The sample is glued, with the help of 3M DP190 Scotch-Weld Epoxy Adhesive, to the sample flange. The glued area is pressed, as required, by the compression flange during the drying of the epoxy.
Fig. 2 Drum method sample holder design scheme

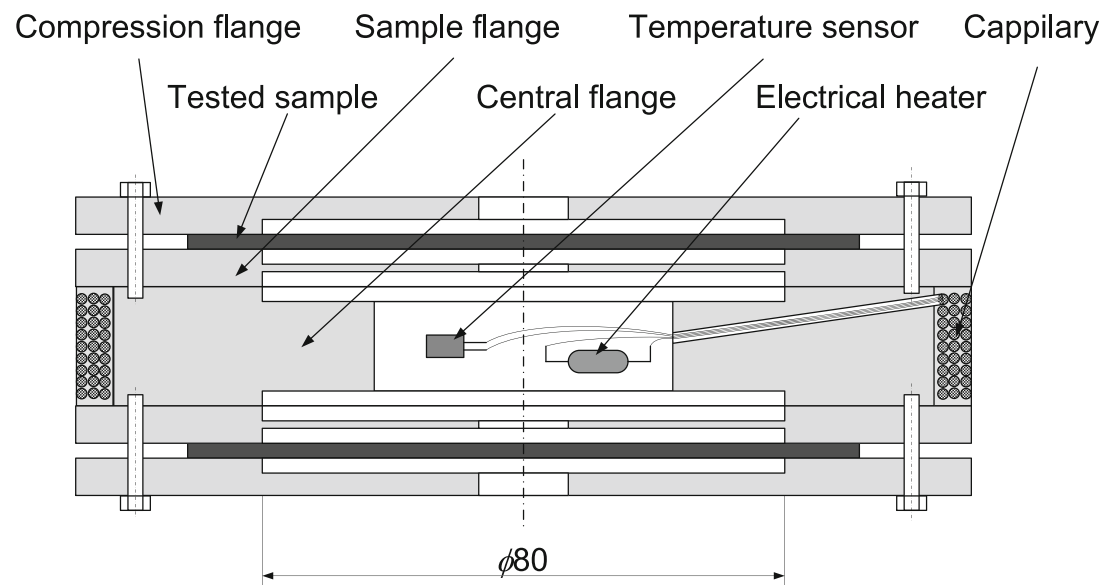


Fig. 3 Total thermal resistance $R_{S}$ in function of $\Delta T$ for sample thickness $0.145 \mathrm{~mm} . T_{b}=1.7 \mathrm{~K}$

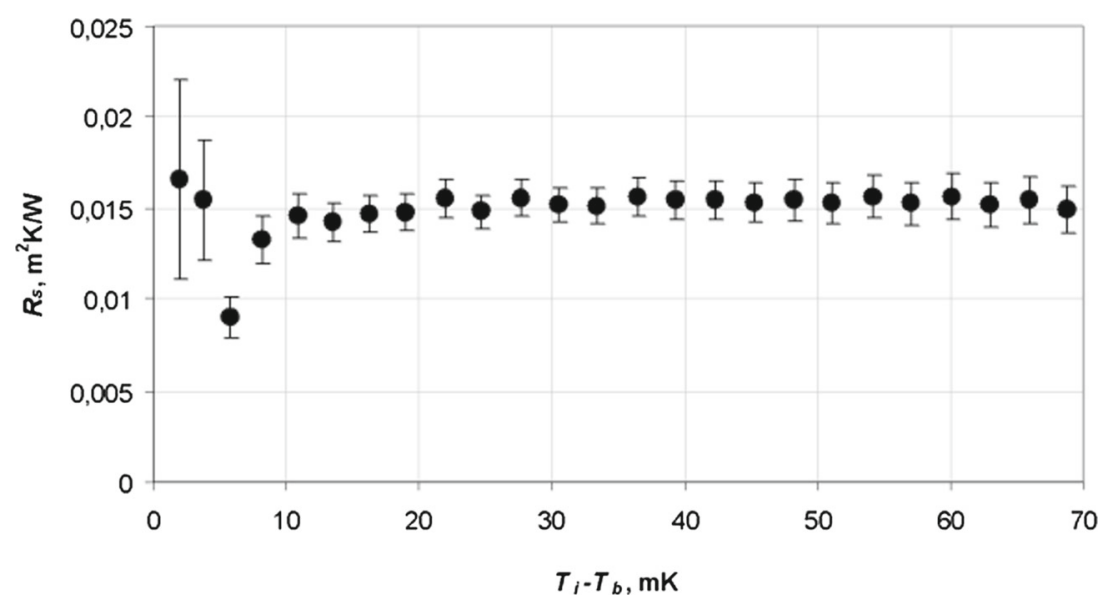

The active heat transfer area of the sample is limited by an $80-\mathrm{mm}$ diameter circle. Two separate samples with this same thickness are installed on the central flange at once. The assembled holder creates the heated (inner) volume of the drum method, limited by the tested material and the central flange material. This volume is filled with HeIIp from the surrounded bath (isothermal volume of the drum method) with the help of a 500-mm long capillary with a $0.75-\mathrm{mm}$ inner diameter, which ensures the minimization of heat loss due to heat conduction through the HeIIp. The capillary also carries $12 \quad 0.2-\mathrm{mm}$ diameter instrumentation wires, four for the temperature sensor, four for the heater, and four for spear wires, which create a further limitation in area for HeIIp heat conduction.

\subsection{Test and Data Fitting Procedure}

In order to determine the Kapitza resistance and thermal conductivity of the tested materials, a superfluid helium cryostat from the Wroclaw University of Technology was used. Three different thicknesses of the LARP material sheet were tested: $145 \pm 7,250 \pm 6$, and $367 \pm 11 \mu \mathrm{m}$. The sample holders were placed inside the cryostat measurement vessel, where the HeIIp bath temperature $\left(T_{b}\right)$ was controlled at a constant level. The total $T_{b}$ error, accounting for the electronic readout and sensor-characteristic fitting errors, is assumed as $\pm 1.0 \mathrm{mK}$, while the $T_{b}$ stabilization was better than $\pm 0.25 \mathrm{mK}$. Materials were tested at seven different $T_{b}$ levels in the range of $1.5-2.0 \mathrm{~K}$. At the given bath temperature, heat $Q$ dissipated
Fig. 4 Total error of Rs value in function of the $\Delta T$

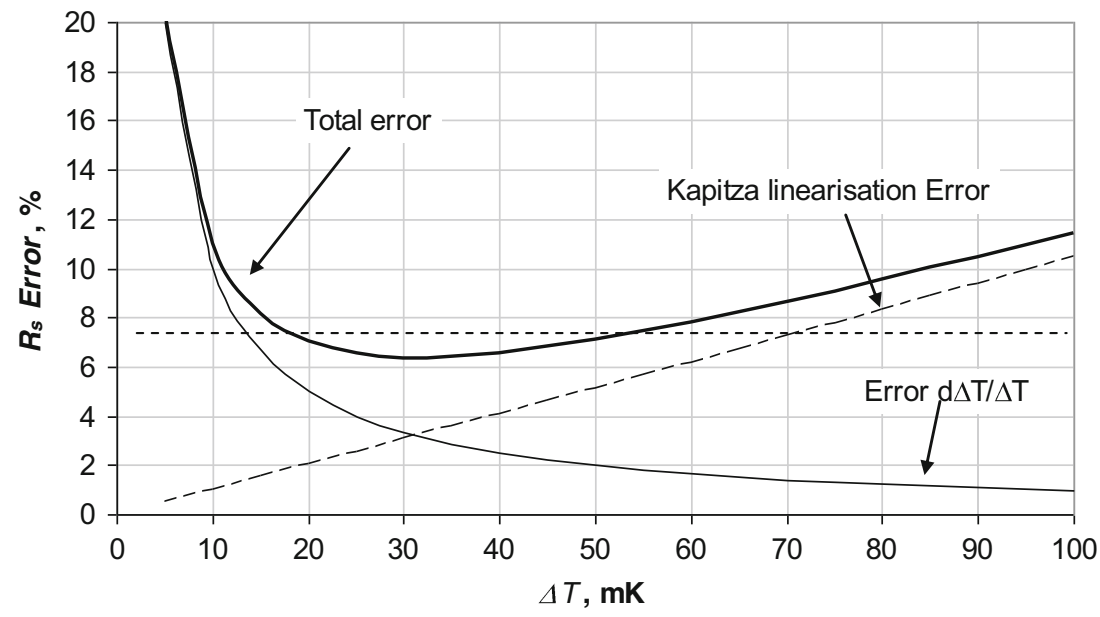


Fig. 5 Total thermal resistance in function of the sample thickness at $T_{b}=1.7 \mathrm{~K}$

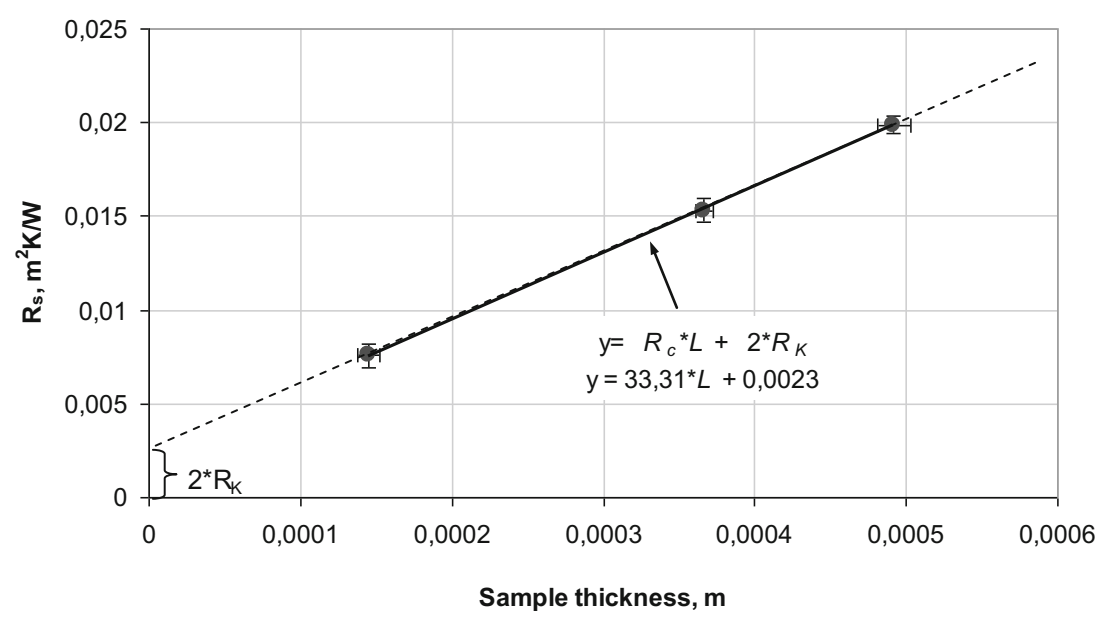

inside each holder in a range of $0-70 \mathrm{~mW}$ with a $2-\mathrm{mW}$ ramp. The heat was generated with the use of the KEITHLEY 2400 source meter with an accuracy better than $0.1 \%$ of the heat value. The temperature of the inner volume $T_{i}$ was recorded in steady state conditions $\left(T_{i}\right.$ stabilization better than $0.25 \mathrm{mK}$ ) at a given value of $Q$. The total error for the $\Delta T$ value, determined as $T_{i}$ at $Q=0$ and $T_{i}$ at the given $Q$ value, can be assumed as $\pm 0.5 \mathrm{mK}$.

The $Q$ transferred from the sample holder inner volume to the HeIIs bath is the sum of the heat transferred through the sample material $Q_{s}$, through the holder material $Q_{\text {hold }}$ and through the capillary $Q_{\text {cap }}$. The $Q_{\text {hold }}$ calculations, performed with the FE method, show that it varies from $2.5 \%$ of $Q$ for $145-\mu$ m thick sample to $4.5 \%$ of $Q$ for $367 \mathrm{~m}$. The capillary heat loss was calculated for each $\Delta T$ value and accounted for in further analysis with an assumed precision of $\pm 20 \%$, as an exact capillary cross-section area for helium conductance is difficult to determine.
Figure 3 presents an exemplary plot of the total thermal resistance $R_{S}$ values in function of $\Delta T$, where the $R_{S}$ is calculated in accordance to (4). A detailed analysis of the total error composition shows that it is dominated by $\Delta T$ error, as well as error resulting from the linearization of the (1), which can be found from (4):

$\varepsilon_{\text {Lin }}=1+\frac{3}{2} \frac{\Delta T}{T_{i}}+\left(\frac{\Delta T}{T_{i}}\right)^{2}+\frac{1}{4}\left(\frac{\Delta T}{T_{i}}\right)^{3}$

The sum of the remaining errors is comparatively small and can be considered to be systematic error. As seen from Figs. 3 and 4, the $R_{S}$ error value is large for $\Delta T<15 \mathrm{mK}$ and for $\Delta T>80 \mathrm{mK}$. In order to minimize total error, the average value of $R_{S}$ with the average $R_{s}$ error values from the range of $20 \mathrm{mK}>\Delta T 50 \mathrm{mK}$ is taken for further analysis.

The next step of the analysis is the preparation of the $R_{S}-$ sample thickness graph for the given $T_{b}$ level-see Fig. 5 . In this graph, $R_{S}$ points, fitting the line slope, stand for
Fig. 6 Thermal conductivity variation with temperature

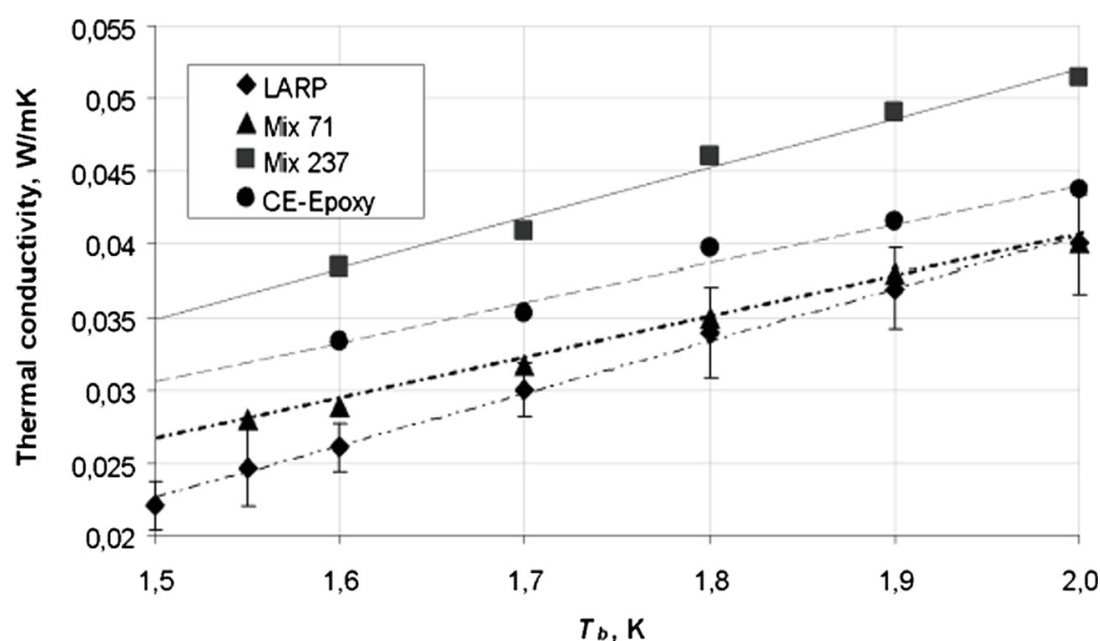


Fig. 7 Kapitza resistance variation with temperature

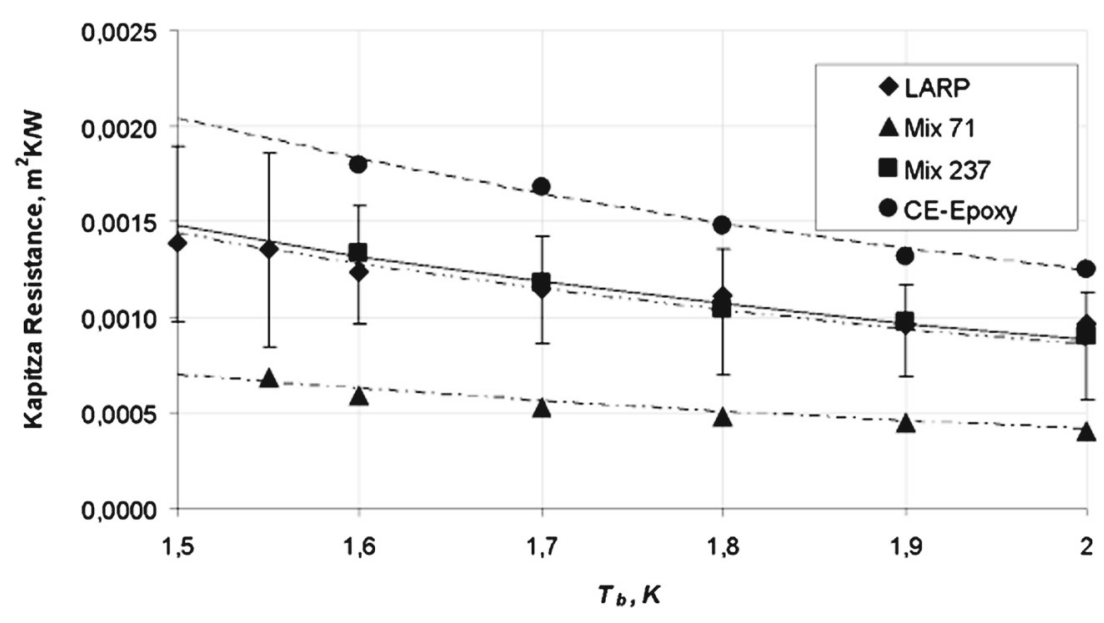

thermal conductance resistance $R_{c}$, while the intersection of the fitting line and $R_{S}$ axis determines the double Kapitza resistance $R_{K}$ value-compare with (3).

\section{Results and Discussion}

In Figs. 6 and 7, thermal conductivity and the Kapitza resistance value in the function of $T_{b}$ in comparison with data obtained for Mix 71, Mix 237, and CE-epoxy materials are presented. It can be found that the LARP material is characterized by the lowest thermal conductivity: 25-35\% lower than Mix 237 and 10-30 \% lower than CE-epoxy. On the other hand, the Kapitza resistance is very similar to the value for Mix 237 but $25 \%$ lower than for CE-epoxy.
Acknowledgments This research has been co-funded by the EC within the EuCARD project (Grant Agreement No. 22757) and by the Polish Ministry of Science and Higher Education statutory funds.

Open Access This article is distributed under the terms of the Creative Commons Attribution License which permits any use, distribution, and reproduction in any medium, provided the original author(s) and the source are credited.

\section{References}

1. Baudouy, B., Polinski, J.: Thermal conductivity and Kapitza resistance of epoxy resin fiberglass tape at superfluid helium temperature. Cryogenics 49, 138-1433 (2009)

2. Pietrowicz, S., Four, A., Jones, S., Canfer, S., Baudouy, B.: Thermal conductivity and Kapitza resistance of cyanate ester epoxy mix and tri-functional epoxy electrical insulations at superfluid helium temperature. Cryogenics 52, 100-104 (2012) 